\title{
ON THE CONVERGENCE OF FOURIER SERIES
}

\section{BY WILLIAM RANDELS}

The purpose of this note is to show that a criterion for the convergence of Fourier series, given by Tonelli, ${ }^{*}$ is contained in the Lebesgue criterion.

The conditions of the Tonelli criterion aref

$$
\phi(t)=o(1) \text { as } t \rightarrow 0 ;
$$

(2) $\phi(t)$ is absolutely continuous in the interval $(\epsilon, \pi), \epsilon>0$, or

$$
\phi(t)=\int_{t_{1}}^{t} \phi^{\prime}(\tau) d \tau, \text { for } t>0 ;
$$

(3) meas. $\lim _{t \rightarrow 0} t \phi^{\prime}(t) \geqq 0$.

Here by meas. lim we mean that in calculating the limit we are permitted to leave out of consideration sets of measure zero. We have to show that, if the above conditions are satisfied, the conditions of the Lebesgue criterion,

$$
\begin{gathered}
\Phi(t)=\int_{0}^{t}|\phi(\tau)| d \tau=o(t), \\
\int_{\delta}^{a}|\phi(t+\delta)-\phi(t)| \frac{d t}{t}=o(1), \text { as } \delta \rightarrow 0, \delta>0,
\end{gathered}
$$

are also satisfied.

Condition (4) is an obvious consequence of (1). Let $\phi^{\prime}(t)=$ $\phi_{1}^{\prime}(t)+\phi_{2}^{\prime}(t)$, where

$\phi_{1}^{\prime}(t)=\left\{\begin{array}{l}\phi^{\prime}(t), t \subset E \equiv E\left(\phi^{\prime}(t) \geqq 0\right), \\ 0, t \subset C(E),\end{array} \phi_{2}^{\prime}(t)=\left\{\begin{array}{l}0, t \subset E, \\ \phi^{\prime}(t), t \subset C(E) .\end{array}\right.\right.$

Then $\left|\phi^{\prime}(t)\right|=\phi_{1}^{\prime}(t)-\phi_{2}^{\prime}(t)=\phi^{\prime}(t)-2 \phi_{2}^{\prime}(t)$. Since $\phi_{2}^{\prime}(t)<0$, condition (3) implies that we have meas. $\lim _{t \rightarrow 0} t \phi_{2}^{\prime}(t)=0$, or $\phi_{2}^{\prime}(t)=o(1 / t)$, except for a set of measure zero. Since we know that $\delta>0$ and $\phi(t)=\int_{t_{1}}^{t} \phi^{\prime}(\tau) d \tau$ if $t>0$,

* L. Tonelli, Serie Trigonometriche, 1928, p. 291.

$\dagger \phi(t)$ is defined in the usual way by $\phi(t)=f(x+t)+f(x-t)-2 f(x)$. 


$$
\begin{aligned}
& \int_{\delta}^{a}|\phi(t+\delta)-\phi(t)| \frac{d t}{t} \\
= & \int_{\delta}^{a} \frac{d t}{t}\left|\int_{t}^{t+\delta} \phi^{\prime}(\tau) d \tau\right| \leqq \int_{\delta}^{a} \frac{d t}{t} \int_{t}^{t+\delta}\left|\phi^{\prime}(\tau)\right| d \tau=I .
\end{aligned}
$$

By the Fubini theorem the order of integration may be interchanged, and as a result,

$$
\begin{aligned}
I= & \int_{\delta}^{2 \delta}\left|\phi^{\prime}(\tau)\right| d \tau \int_{\delta}^{\tau} \frac{d t}{t}+\int_{2 \delta}^{a}\left|\phi^{\prime}(\tau)\right| d \tau \int_{\tau-\delta}^{\tau} \frac{d t}{t} \\
& +\int_{a}^{a+\delta}\left|\phi^{\prime}(\tau)\right| d \tau \int_{\tau-\delta}^{a} \frac{d t}{t} \\
= & \int_{\delta}^{2 \delta}\left|\phi^{\prime}(\tau)\right| \log \frac{\tau}{\delta} d \tau+\int_{2 \delta}^{a}\left|\phi^{\prime}(\tau)\right| \log \frac{\tau}{\tau-\delta} d \tau \\
& +\int_{a}^{a+\delta}\left|\phi^{\prime}(\tau)\right| \log \frac{a}{\tau-\delta} d \tau=I_{1}+I_{2}+I_{3} .
\end{aligned}
$$

We assume now that $a$ is taken sufficiently small; then

$$
\begin{aligned}
I_{1} & \leqq \log 2 \int_{\delta}^{2 \delta}\left|\phi^{\prime}(\tau)\right| d \tau=\log 2\left(\int_{\delta}^{2 \delta} \phi^{\prime}(\tau) d \tau-2 \int_{\delta}^{2 \delta} \phi_{2}^{\prime}(\tau) d \tau\right) \\
& =o\left(1+\int_{\delta}^{2 \delta} \frac{d \tau}{\tau}\right)=o(1), \\
I_{2} & =\int_{2 \delta}^{a} \phi^{\prime}(\tau) \log \frac{\tau}{\tau-\delta} d \tau-2 \int_{2 \delta}^{a} \phi_{2}^{\prime}(\tau) \log \frac{\tau}{\tau-\delta} d \tau=I_{2}^{\prime}+2 I_{2}^{\prime \prime}, \\
I_{2}^{\prime} & \left.=\phi(\tau) \log \frac{\tau}{\tau-\delta}\right]_{2 \delta}^{a}+\delta \int_{2 \delta}^{a} \phi(\tau) \frac{d \tau}{\tau(\tau-\delta)} \\
& =o\left(1+\delta \int_{\delta}^{a} \frac{d \tau}{\tau^{2}}\right)=o(1), \\
I_{2}^{\prime \prime \prime} & =-\int_{2 \delta}^{a} \phi_{2}^{\prime}(\tau) \log \frac{\tau}{\tau-\delta} d \tau=\int_{2 \delta}^{a} \phi_{2}^{\prime}(\tau) \log \frac{\tau-\delta}{\tau} d \tau \\
& =o\left(\int_{2 \delta}^{a} \log \left(1-\frac{\delta}{\tau}\right) \mid \frac{d \tau}{\tau}\right),
\end{aligned}
$$




$$
\begin{gathered}
\int_{2 \delta}^{a}\left|\log \left(1-\frac{\delta}{\tau}\right)\right| \frac{d \tau}{\tau}=-\int_{1 / 2}^{\delta / a}|\log (1-\tau)| \frac{d \tau}{\tau} \\
=\int_{\delta / a}^{1 / 2}|\log (1-\tau)| \frac{d \tau}{\tau}=o(1) .
\end{gathered}
$$

Hence $I_{2}=o(1)$. Finally it is obvious that $I_{3}=o(1)$ as $\delta \rightarrow 0$. On combining these facts, we obtain the desired result:

$$
\int_{\delta}^{a}|\phi(t+\delta)-\phi(t)| \frac{d t}{t}=o(1) .
$$

BROWN UNIVERSITY

\section{A NOTE ON COMPACTNESS*}

BY E. H. HANSON

The purpose of this note is to deduce the conditions for compactness $\dagger$ of a set of measurable functions from the general criterion for compactness in complete metric spaces. $\ddagger$ This is the procedure that suggests itself immediately and it suceeds without any difficulty. The general criterion referred to asserts that a set $S$ of elements of a complete metric space is compact if and only if, for every positive $\epsilon, S$ is inclosable in a finite number of spheres $\S$ of radius $\epsilon$; and the validity of this the reader may easily verify for himself. Fréchet $\|$ has obtained the result for

* Presented to the Society, February 25, 1933. I wish to express my gratitude to Professor Henry Blumberg for suggesting the idea of this note and for helpful criticism during its preparation.

$\dagger$ A set $S$ of elements of a space is compact if every infinite subset of $S$ has at least one limit point in the space.

$\ddagger$ A space $M$ is said to be metric if there exists a positive or zero function $d\left(e_{1}, e_{2}\right)$ of pairs of elements of $M$ satisfying the conditions: (1) $d\left(e_{1}, e_{2}\right)=d\left(e_{2}, e_{1}\right)$, (2) $d\left(e_{1}, e_{2}\right)=0$ is equivalent to $e_{1}=e_{2} ;(3) d\left(e_{1}, e_{3}\right) \leqq d\left(e_{1}, e_{2}\right)+d\left(e_{2}, e_{3}\right)$. A metric space is complete if $\lim _{m, n \rightarrow \infty} d\left(e_{m}, e_{n}\right)=0$ implies the existence of an element $e$ such that $\lim _{n \rightarrow \infty} d\left(e_{n}, e\right)=0$.

$\S$ A sphere with center $c$ and radius $r$ is by definition the set of elements $e$ of $M$ such that $d(e, c)<r$.

$\|$ Sur les ensembles compacts de fonctions mesurables, Fundamenta Mathematicae, vol. 9 , p. 25. 